\title{
Effects of overt and covert task instructions and stimulus modality on orienting response recorded by electrodermal indices ${ }^{1}$
}

\author{
AKIRA IMAI ${ }^{2}$ \\ Department of Psychology, School of Letters, Nagoya University, Chikusa-ku, Nogoya 464-01
}

\begin{abstract}
Two experiments were conducted to examine the occurrences of "selective" orienting response (OR) and "alcrtive" OR by electrodermal response (EDR) in connection with task instructions and stimulus modalities. Two groups of 36 female university students reccived two visual and/or auditory stimuli during both habituation and test sessions either unimodally (Exp. I) or bimodally (Exp. II). The subjects in both experiments were assigned to onc of three subgroups: PR (pedal-responding), SC (stimulation-counting), and NI (neutral-instruction). The PR was instructed to pedal to the test stimulus; the SC to count the number of the test stimulus presentations; the NI to do nothing. Results are as follows: (1) In Exp. I, a significant increase of the EDR to the test stimulus (selective OR) was observed by both the PR and the SC. (2) In Exp. II, only the PR alone showed significant augmental changes in the EDR magnitude to the non-test stimuli (alertive OR) as well as to the test stimuli. It was suggested, therefore, that the covert task had a little possibility for eliciting the alcrtive OR.
\end{abstract}

Key words: orienting response (OR), electrodermal response (EDR), overt task, covert task, selective $O R$, alcrtive $O R$.

A task instruction to a stimulus generally makes that stimulus more significant. Under experimental situations, two kinds of tasks manipulate the significance of the stimulus: One is the requirement to respond overtly to the stimulus, for instance, by pressing a key or a pedal, grasping a hand, or producing a verbal response; the other is the requirement to pay attention covertly to the stimulus, for example, through free-association, counting, or making a judgement.

The instruction to perform an overt

1 Part of this research was presented at 52nd annual convention of the Japanese Psychological Association, October, 1988, Hiroshima. This research was also supported partly by a Grant-inAid for Scientific Researches (No. 01790030; 1989), Ministry of Education, Science and Culture of Japan.

2 The author makes a grateful acknowledgment to Professor Takuo Goto, Department of Psychology, School of Letters, Nagoya University for his continuing guidance and encouragement on this research task generates a greater magnitude of orienting response (OR) measured by electrodermal response (EDR) than those instructions without any task, overt or covert (Germana, 1968; Harding \& Punzo, 1971; Imai, 1988; Maltzman \& Raskin, 1979; Ray, Piroch, \& Kimmel, 1977; Sokolov, 1963). In contrast, the effects of the covert task show contradictory tendencies. For instance, Maltzman and Raskin (1979) indicated that subjects exerting covert responses to a word stimulus were more responsive in the EDR than the control group which was given no task.

However, Badia and Harley (1970) failed to confirm the effects of the instruction on the EDR responsivity when judging the shock intensity. Moreover, Becker and Shapiro (1980) found that the EDR magnitudes of the covert task (paying attention to and counting the click stimuli) were not different from those of the control task (ignoring the click stimuli and maintaining a state of relaxa- 
tion). Similar results were obtained by Harding and Punzo (1971) and Orlebeke and Passchier (1976). In addition, a requirement to ignore the stimulus also resulted in contradictory tendencies: an increase in EDR responsivity (Kohlenberg, 1970) and a decrease in EDR responsivity to the ignored stimulus (Iacono \& Lykken, 1983, 1984).

In an attempt to clear up the complicated findings and in order to investigate the effects of the covert task on the significance of the stimulus, it seems reasonable to examine the difference between the overt and the covert task requirements under an identical experimental situation. In this experiment, it is necessary to confirm that the subjects perform the covert task correctly. If done strictly, the effects of the overt and the covert tasks should result in the same tendencies if these tasks have the same nature in response to the stimuli.

The first purpose of the present study is to examine the effects of the overt and the covert tasks on the EDR-OR. In this study, two tasks were applied: (1) pressing a pedal as an overt response, and (2) counting the frequency of stimulus appearances as a covert one.

The second purpose is to specify under which condition the "alertive OR" (Imai, 1988) would occur. In previous experiments using simple figures and tones (Imai, 1988), I confirmed the "selective OR" (Maltzman \& Raskin, 1979) to the significant stimulus when the significant and the non-significant stimuli were presented unimodally. Moreover, I found that an increase in $O R$ was generated by the non-significant stimulus when the significant and the non-significant stimuli were provided bimodally. I named this OR as " alertive OR". However, further investigation is necessary to clarify the condition under which this alertive OR will be elicited.

For these two purposes, two experiments are conducted in this study. The signif- icant stimulus and the non-significant stimulus are presented unimodally in experiment $\mathrm{I}$, and bimodally in experiment II. Through these experiments, the effects of the overt and the covert tasks on the OR are cxamined, and the following hypotheses are tested: (1) The overt task will croke the selective OR to the significant stimulus when the significant and the non-significant stimuli are presented unimodally. In addition, the selective OR will also be elicited according to the degree of significance imparted by the covert task. (2) The alertive OR to the non-significant stimulus will emerge as a consequence of overt activity when the significant and the non-significant stimuli are presented bimodally. Moreover, the alertive $O R$ will also occur according to the degree of significance imparted by the covert task.

\section{Experiment I}

The purpose of this experiment is to examine the selective OR under the overt and the covert tasks by using the significant and the non-significant stimuli in the identical modality.

\section{Methods}

Subjects. Subjects were 36 female university students. Their ages ranged from 18 to 23 . They were assigned equally and randomly to one of three groups.

Apparatus. The EDR in DC. recording was obtained through NIHON $\mathrm{KOH}$ DEN Ag-AgCl biopotential electrodes attached to the palm and the ventral side of the subject's left forearm with standard electrode paste, by use of a NIHON KOHDEN GSR-2100 bridge box. This EDR was recorded on a NEC-SANEI 8K31 two-channel penoscillograph with chart speed set at $1 \mathrm{~mm} / \mathrm{s}$.

Material. Two stimulus series (unimodal presentation) were applied by using visual or auditory stimuli. Figure 1 shows the two stimulus figures (nonsense pat- 
terns) used in the experiments. They appeared on a NEC computer (PC-8801) CRT display in a visual angle of five degrees in width and with a $500 \mathrm{~ms}$ duration. The two auditory stimuli were low intensity of $54 \mathrm{~dB}(\mathrm{C})$ beep tones $(2400 \mathrm{~Hz})$ delivered by the above computer. One tone was presented for a duration of $500 \mathrm{~ms}$, and the other was split into five segments and presented for a duration of $100 \mathrm{~ms}$ per segment with a $30 \mathrm{~ms}$ interval between segments. The above computer controlled the stimulus presentation and the time schedule.

Procedure. The two experiments had sessions of habituation and test. Subjects were informed that the purpose of this study was to measure the changes in the electrical resistance of the skin by monotonous stimulation and that the expeirment with two sessions would take about 20 minutes. Before the habituation session, half of the subjects were instructed to look at the figures when they appeared on the CRT, and the other half was told to listen to the tones. After a three minute rest period, the habituation session started. The habituation series consisted of 20 presentations in which two kinds of stimulus appeared 10 times each in random order. The interstimulus intervals ranged from 15 to $25 \mathrm{~s}$ with an average of $20 \mathrm{~s}$. After the habituation session, the subjects received one of the following three instructions: (1) Pedal-responding group (PR) was
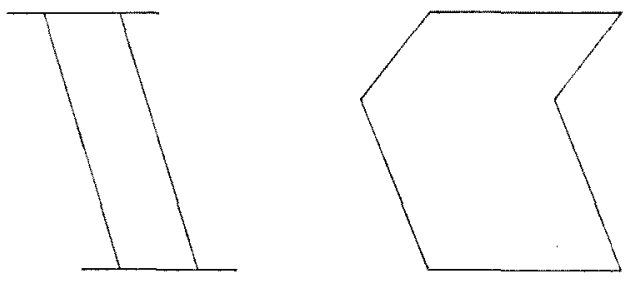

Fig. 1. Visual stimuli applied in the experiments. These stimuli were sclected from 32 figures used by Teraoka (1971) in his study on concept learning, because of their simplicity in being made of straight lines. informed to pedal as quickly as possible when one (test stimulus) of two figures (or tones) of the habituation stimulus appeared. (2) Stimulation-counting group (SC) was asked to count the number of presentations of the test stimulus. (3) Neutral-instruction group (NI) was given no additional instructions. Then, the test session started in the same manner as in the habituation session except for the number of stimulations, which were reduced from 20 to 12 (each presented six times). After the test session the subjects in the $\mathrm{SC}$ reported the frequency of their counting. The one subject who didn't produce a correct counting was replaced.

Scoring. The largest resistance change occurring within the interval of $0.5-5.0 \mathrm{~s}$ following the stimulus onset was recorded as the EDR. Conductance change was obtained by the following formula: $\Delta C$ (unit: $\mu S)=\left(1 / R^{\prime}-1 / R\right) \times 1000$, where $R$ is resistance $(K \Omega)$ at the onset, and $R^{\prime}$ is the resistance at the peak of response. Then, the data of error trial in pedal response of the PR were taken off as an artifact. In addition, $1 / R \times 1000$ was defined as the electrodermal level (EDL), and $1 / R$ for the onset of the test stimulus was discarded in the analysis. Thus, twenty EDL scores were collected at the habituation session and six at the test session. These EDR and EDL scores were averaged over twotrial blocks, and $\log$ transformation was introduced to all scores for statistical analysis.

\section{Results and Discussion}

There was no significant difference in the EDR magnitudes between visual and auditory stimulations during the habituation session by the analysis of variance (ANOVA). Then, the magnitudes of visual and auditory stimulation were combined together for the resulting analyses. Moreover, adjustments of conservative degree of freedom to the repeated measures tests recommended by Greenhouse and Geisser (1959) were applied to the analy- 
sis of the EDL, because the variancecovariance matrix of the EDL was not homogeneous.

Habiluation session. "HABITUATION" in Fig. 2 shows the mean amplitudes of the EDR for the three groups plotted as two-trial blocks, These groups did not differ in the response magnitudes, the tendencies of response decrement, and the initial responsivity. The ANOVA with repeated measures revealed that there was only a significant main effect for blocks $\left(F_{(9,297)}=8.21, p<.001\right)$. No other significant differences were found.

The ANOVA with repeated measures of the EDL indicated the significant main effect for blocks $\left(F_{(1,38)}=6.15, p<.05\right)$, which revealed the tendency of the EDL to decrease gradually over groups. No other significant effects were obtained.

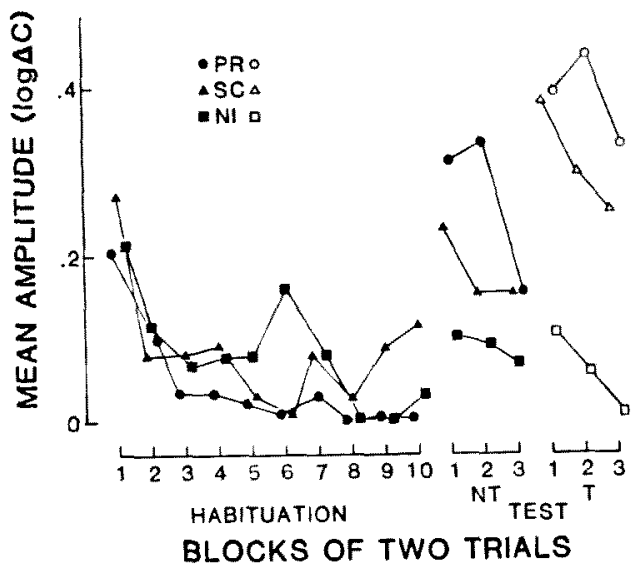

Fig. 2. Mean amplitude of the EDR for the threc groups of PR (pedal-responding), SC (stimulation-counting), and NI (neutral-instruction) plotted for two-trial blocks in Exp. 1. "HABITUATION" shows the magnitudes of response obtained during the habituation session. At "TEST. NT", the response magnitudes to the non-significant stimulus (NT) during the test session are shown by three kinds of filled marks. The response magnitudes generated by the significant stimulus ( $T$ ) are indicated by the same three empty marks at "TEST-T". Circles represent responses of the $\mathrm{PR}$, triangles those of the $\mathrm{SC}$, and squares those of the NI.
Test session. "TEST'N'I'" in l'ig. 2 illustrates the mean amplitudes of the EDR for three groups to the non-lest stimulus (NT) plotted as the two-trial blocks. The response magnitudes suggested the tendency of increased responsivity in the order of PR, SC, and NI. However, the ANOVA with repeated measures showed no significant group difference and no significant group-by-block interaction. Only a main effect of block was significant $\left(F_{(2,68)}=3.88, p<.05\right)$.

Meanwhile, "TEST-T" in Fig. 2 indicates the mean amplitudes of the EDR to the test stimulus ( $T$ ) in the same manner as the above "TEST-NT". $\mathrm{Al}$ though responsivity for the $\mathrm{T}$ showed the same tendency as for the NT, the task groups of $\mathrm{PR}$ and $\mathrm{SC}$ are different from the NI group in their magnitudes. Namely, the magnitudes of $\mathrm{PR}$ and $\mathrm{SG}$ for the $\mathrm{T}$ are greater than for the NT. The ANOVA with repeated measures confirmed this tendency in terms of the significant main effects of the group $\left(F_{(2,33)}=4.22, p<.05\right)$ and the block $\left(F_{(2,66)}=3.82, p<.05\right)$ without their interaction. Further analysis by Ryan's multiple comparison method revealed that the $P R$ differed significantly from the NI $\left(t_{(33)}=2.78, p<.05\right)$, but not from the $\mathrm{SC}$. In addition, there was a marginal difference between the $\mathrm{SC}$ and the NI $\left(t_{(33)}=2.13, p<.10\right)$.

In order to compare the difference between the EDR magnitudes to the NT and to the $\mathrm{T}$ during the test session, three separate ANOVAs were applied. The magnitudes to the $T$ were significantly larger than those of the NT in the PR $\left(F_{(1,11)}=5.83, p<.05\right)$ and also in the $\mathrm{SC}$ $\left(F_{(1,1)}=5.34, \quad p<.05\right)$. A main block effect was also significant for the PR $\left(F_{(2,22)}=4.16, p<.05\right)$, and a marginal effect of block was found for the $\mathrm{SC}$ $\left(F_{(2,22)}=2.76, p<.10\right)$. For the NI, neither main effects nor interaction were obtained.

On the other hand, the ANOVA of the EDL with repeated measures indicated 
that only a main block effect was significant $\left(F_{\{1,33)}=5.23, p<.05\right)$ in revealing the response decrement across the blocks.

In the present experiment, there was no difference in the magnitudes of the EDR to the NT among the three groups of the $\mathrm{PR}$, the SC, and the NI, while the largest magnitude of the EDR to the $T$ was found in the PR. In addition, the SC showed a larger EDR to the $T$ than the NI did. Though these tendencies supported the previous finding that the selective OR emerged only for the significant stimulus presented unimodally (Maltzman \& Raskin, 1979; Imai, 1988), this experiment indicated that the task effects were clearer for the PR than for the SC.

\section{Experiment II}

The purpose of this experiment is to examine the alertive OR generated by the non-significant stimulus under the overt and the covert tasks by using the significant and the non-significant stimuli in the different modality.

\section{Methods}

Subjects and material. Subjects were 36 female university students aged from 18 to 21 and they were different from those in Exp. I. The subjects were assigned to one of the three groups in the same manner as in Exp. I. Four stimuli employed in Exp. I were also applied in this experiment. These stimuli were combined into four different stimulus series consisting of two different kinds of modality.

Procedure. Almost the same procedure as in Exp. I was employed except for the following three points: (1) Before the habituation session, the subjects were required to look at the visual stimulus on the CRT display and to listen to the auditory one delivered by the front computer. (2) During the habituation session, both kinds of stimulus were presented 10 times equally and randomly. (3) During the test session, both kinds of stimulus were presented six times in random order.

\section{Results and Discussion}

There was no significant difference in the EDR magnitudes among the four stimulus series during the habituation session by the ANOVA. Since the variancecovariance matrix was not homogeneous for the EDR and the EDL in this experiment, Greenhouse and Geisser conservative test (1959) were also applied to these data.

Habituation session. "HABITUATION" in Fig. 3 shows mean amplitudes of the EDR during the habituation session plotted in the same way as in Fig. 2. In the ANOVA to the EDR, a significant main effect of block $\left(F_{(1,33)}=8.44, p<.01\right)$ represented the tendency toward the habituation of response.

Only a main effect of block was significant in the ANOVA of the EDL $\left(F_{(1,33)}\right.$ $=8.63, p<.01)$, and this significant effect suggested the decrement in the EDL across the blocks in common with the above magnitudes of EDR.

Test session. "TEST-NT" in Fig. 3 represents mean amplitudes of the EDR to the NT plotted in the same manner as

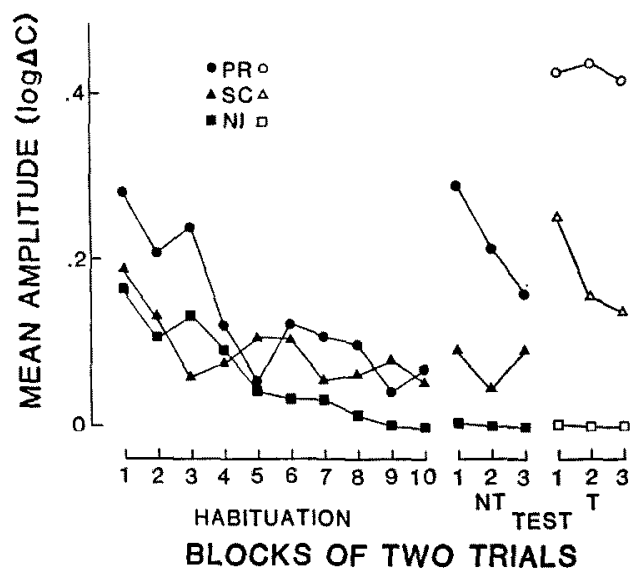

Fig. 3. Mean amplitude of EDR obtained in Exp. II and plotted in the same way as in Fig. 2. Other conventions are identical with those in Fig. 2. 
in Fig. 2. The ANOVA on the EDR to the NT showed a significant main effect of group $\left(F_{(2,3)}=7.76, p<.01\right)$. No other significant effects were obtained. Further analysis by Ryan's multiple comparison method indicated the significant differences between the $P R$ and the NI $\left(t_{(93)}=3.80, \quad p<.01\right)$, and also between the PR and the SC $\left(t_{(s)}=2.79, p<.05\right)$. However, no significant difference was found between the SC and the NI.

"TEST $-T$ " in Fig. 3 illustrates mean amplitudes of the EDR to the $T$ plotted in the same way as in Fig. 2. As shown in the figure, the largest magnitudes of the EDR were indicated for the $P R$, the magnitudes of the EDR for the NI were smallest, and the magnitudes for the $\mathrm{SC}$ lay between the PR and the NI. The ANOVA on the EDR to the $T$ showed a significant main effect of group $\left(F_{(2,33)}\right.$ $=9.03, p<.001)$. There was also a marginal main effect of block $\left(F_{(1,33)}=3.15\right.$, $p<.10)$. Further analysis by Ryan's multiple comparison method indicated significant differences between the $P R$ and the NI $\left(t_{(33)}=4.22, p<.01\right)$, and between the PR and the SC $\left(t_{(33)}=2.51, p<.05\right)$. There was, however, no difference between the SC and the NI.

In order to compare the differences between the EDR magnitudes to the NT and those to the $T$ as in Exp. I, the separate ANOVA for each group was introduced. For the PR, the EDR magnitudes to the $T$ were significantly larger than those to the NT $\left(F_{(1,1)}=5.83, p<\right.$ $.05)$. For the $\mathrm{SC}$, there were significant main effects of stimulus $\left(F_{(1,1)}=5.67\right.$, $p<.05)$ and block $\left(F_{(2,22)}=3.53, p<.05\right)$. In addition, interaction between these two effects was also significant $\left(F_{(2,22)}=\right.$ $3.53, p<.05)$. The ANOVA on the EDR of the NI group demonstrated no significant effects.

Meanwhile, the EDL decreased across the blocks. This decrement was confirmed through the ANOVA in which only a main effect of block was significant
$\left(F_{(1,38)}=14.90, p<.001\right)$.

In this experiment, the EDR magnitudes to the "significant" stimulus in the $T$ differed significantly among the above three groups. Moreover, there was significant difference in the EDR magnitudes among these groups to the "nonsignificant" stimulus in the NT.

\section{General Discussion}

In Exp. I, the significant stimulus in the $T$ and the non-significant stimulus in the NT were presented in visual and auditory identical modality. The EDR magnitudes to the $\mathrm{T}$ in the $\mathrm{PR}$ and the SCishowed significant difference from those of the NI. In contrast, the EDR magnitudes to the NT did not differ statistically among the three groups (PR, SC, and NI). Though the EDR magnitude of the NI to the NT at the first block of test session scems to be higher than that at the tenth block of habituation session, this difference was not significant by the t-test. Accordingly, the insignificant diflerence in the NT EDR magnitudes among the three groups should not be regarded as the increased responsivity of the NI group.

These tendencies suggest that the "sclective OR" occurred here to the significant stimulus when the significant and the non-significant stimuli were presented unimodally. These results in Exp. I supported the above-mentioned hypothesis (1), and agreed with the results of previous studies (Maltzman \& Raskin, 1979; Imai, 1988).

While, in Exp. II, when these stimuli were presented bimodally, there were significant differences in the EDR rcsponsivity among the three groups both to the $T$ and to the NT. This result suggests the occurrence of the "alertive OR " to the non-significant stimulus when bimodal presentations were applied to the significant and the non-significant stimuli (Imai, 1988). However, this tendency 
was supported only by the PR. The EDR magnitudes to the NT in the SC did not differ from those in the NI. Thus, only the former part of the abovementioned hypothesis (2) was confirmed in this study.

As to the task effects, the overt and the covert response requirements demonstrated differences in relation to the stimulus modality. In Exp. I, the PR and the SC showed more responsivity to the $T$ than the NI, although the increased responsivity was less in the $\mathrm{SG}$ than in the PR. In Exp. II, however, the same comparison as in Exp. I indicated no reliable difference between the $\mathrm{SC}$ and the NI.

The results of the present study for the subjects in the $\mathrm{SC}$ (required covert task) indicate the response tendency as follows: (1) When the significant and the non-significant stimuli are presented unimodally (Exp. I), these stimuli are discriminated from each other strictly, and responsivity to the significant stimulus increases more selectively. (2) If these stimuli were applied bimodally (Exp. II), stimulus discrimination may be generated in the minimum degree. Consequently, the change in stimulus modality may cause a decrease in the difficulty of the discriminative task during covert activity rather than when doing an overt task. However, it is necessary to confirm this tendency in further research.

For the subjects in the PR, the overt task elicited the "selective OR" to the significant stimulus under the unimodal presentation of the stimuli. Under the bimodal stimulation, however, the overt task showed an increase in the EDR magnitudes to the non-significant stimulus, and this augmental change may be due to the "alertive OR".

The "alertive OR" observed here in the PR and also in the previous study (Imai, 1988) does not coincide with the traditional theories of the OR. At first, the "stimulus comparator theory" pro- posed by Sokolov (1960, 1963) required a "neuronal model of stimulus" which is assumed to be constructed in the cortcx. According to his theory, if there is a mismatch between the neuronal model of stimulus and the presented stimuli, the OR would occur. In the present experiments, however, the stimuli presented in the habituation and the test sessions were identical, and there was no mismatch between these two sessions. Consequently, the stimulus comparator theory is not suitable for explaining the above results in this study.

Secondly, Bernstein (1969) concluded that only if the novel stimuli were judged to be of some significance, the OR would occur to that stimuli. Since there were no novel stimuli in this study, his idea is also not applicable to the present findings. Though the introduction of the overt and the covert tasks in the test session may impart novelty to the test stimuli, the analysis of subjects' introspection after the experiment revealed no possibility of such a novelty generation.

Thirdly, no differences in the EDL were found among the three groups in the present study. At present, the EDL has been regarded as the index of the tonic arousal level of the organism. Hence, the "two process theory" proposed by Groves and Thompson (1970) could also not be applicable to the above findings, because the theory assumes that both habituation and sensitization processes may interact with each other in determining the organism's responsivity. In addition, an incremental change in the sensitization process should heighten the tonic arousal level. As a result, this theory does not agree with the present findings in which there is no difference in the EDL among the three groups.

Conclusions in the present study are as follows; (1) The overt and the covert tasks resulted in the selective OR to the significant stimulus when the significant and the non-significant stimuli were presented 
unimodally. (2) The overt task generated the selective OR in greater degree than the covert task did. (3) The alertive OR was evoked only by the overt lask when the signifcant and the nonsignificant stimuli were applied bimodally. (4) Task instructions of the overt and the covert activities did not show any effects on the arousal level measured by the EDL.

\section{References}

Badia, P. \& Harley, J. P. 1970 Habituation and temporal conditioning as related to shock intensity and its judgment. Journal of Experimental Psychology, 84, 534-536.

Becker, D. E., \& Shapiro, D. 1980 Directing attention toward stimuli affects the P300 but not the orienting response. Psychophysiology, 17, 385-389.

Bernstein, A. S. 1969 To what docs the orienting response respond? Psychophysiology, 6, 338-350.

Germana, J. 1968 Response characteristics and the orienting reflex. Journal of Experimental Psychology, 78, 610-616.

Greenhouse, S.W., \& Gisser, S. 1959 On methods in the analysis of profile data. Psychometrika, 24, 9j-112.

Groves, P. M., \& Thompson, R. F. 1970 Habituation: A dual-process theory. Psyctological Review, 77, 419-450.

Harding, G., \& Punzo, F. 1971 Response uncertainty and skin conductance. Journal of Ex. perimental Psychology, 88, 265-272.

Iacono, W. G., \& Lykken, D. T. 1983 The effects of instructions on electrodermal habitua- tion. Pychophysiology, 20, 71-80.

Jacono, W. G., \& Lykken, D. T. 1984 The offects of instructions and an engaging visual task on habituation to loud tones: An evaluation of an alternative to the traditional habituation paradigm. Physiological Psychology, 12, 23-29.

Imai, A. 1988 Efrects of stimulus modality and task instruction upon the orienting response. Japanese Journal of Psychology, 59, 30-36. (In Japanese with English summary)

Kohlenberg, R.J, 1970 Instructions to ignore a stimulus and the GSR. Psychonomic Srience, 19, 220-221.

Malcman, I., \& Raskin, D. C. 1979 Selective orienting and habituation of the GSR as a consequence of overt and covert activity. Physiological Psychology, 7, 204-208.

Orlebekc, J.F, \& Passchier, J. 1976 Organismic, stimulus and task determinants of phasic and tonic heart rate and skin conductance changes. Biological Psychology, 4, 173-184.

Ray, R. L., Piroch, J. F., \& Kimmel, II. D. 1977 The effect of task and stimulus variability on habituation of electrodermal and vasomotor rcactions. Physiological Psychology, 5, 189-196.

Sokolov, E. N. 1960 Neuronal models and the orienting reflex. In M.A.B. Brazier (Ed.), The central nerous system and behavior. Transactions of the third conference. New York: Josian Macy Jr. Foundation. Pp. 187 276.

Sokolov, E. N. 1963 Perception and the conditioned reflex. Oxford: Pergamon Press.

Teraoka, T. 1971 An experimental analysis of selection strategies in the problem solving. Japanese Journal of Psychology, 42, 24-39. (In Japanese with English summary)

(Received Sept. 14, 1989; accepted May 19, 1990) 